\title{
Influence of AO chain length, droplet size and oil to water ratio on the distribution and on the activity of gallates in fish oil-in-water emulsified systems: Emulsion and nanoemulsion comparison
}

\author{
Marlene Costa $^{\mathrm{a}}$, Sonia Losada-Barreiro ${ }^{\mathrm{a}, \mathrm{b}}$, Carlos Bravo-Díaz ${ }^{\mathrm{b}}$, António A. Vicente ${ }^{\mathrm{c}}$, \\ Luís S. Monteiro ${ }^{\mathrm{d}}$, Fátima Paiva-Martins ${ }^{\mathrm{a} \text {,* }}$ \\ ${ }^{\text {a }}$ REQUIMTE/LAQV, Department of Chemistry and Biochemistry, Faculty of Science, University of Porto, Rua do Campo Alegre 687, Porto, Portugal \\ ${ }^{\mathrm{b}}$ Universidad de Vigo, Facultad de Química, Departamento de Química - Física, 36310 Vigo, Spain \\ ${ }^{\mathrm{c}}$ Centre of Biological Engineering, University of Minho, Campus de Gualtar, 4710-057 Braga, Portugal \\ ${ }^{\mathrm{d}}$ Chemistry Centre, University of Minho, Campus de Gualtar, 4710-057 Braga, Portugal
}

\section{A R T I C L E I N F O}

\section{Keywords:}

Nanoemulsion

Gallic acid

Gallates

Antioxidant

Distribution

Antioxidant activity

\begin{abstract}
A B S T R A C T
The distribution of a homologous series of polyphenol derivatives of increasing lipophilicity has been determined in fish oil-in-water emulsions and nanoemulsions by the pseudophase model. One of the hypotheses on which the pseudophase model is based, is that its application is independent of the size of emulsion droplets. In agreement with our hypothesis, results showed that the smaller droplet size found in nanoemulsions does not affect partition constants of gallic acid (GA) and its esters. The antioxidant efficiency of GA and gallates in the emulsified systems used, correlated positively with the concentration of antioxidant at the interfacial region. The increase in the oil/water ratio increased the overall oxidative stability of emulsions but decreased the antioxidant efficiency of the more lipophilic derivatives. This can be assigned to the fact that, increasing the oil phase volume, the interfacial concentration decreased for the more lipophilic antioxidants.
\end{abstract}

\section{Introduction}

In recent years, there has been a growing interest in the use of nanoemulsions as delivery systems of bioactive compounds and in parenteral nutrition (McClements \& Rao, 2011; McClements, 2018). Consequently, it is important to understand the factors that influence the oxidative stability of these formulations and to increase their safety and shelf life. The prevention of oxidation has particular importance when the oil phase of the emulsified system is fish oil as a consequence of its high degree of lipid unsaturation (McClements, Decker, Park, \& Weiss, 2009). Efforts are being made to minimize the impact of the oxidation of marine oils by controlling oxygen content in packaging, optimizing food processing, and by controlling their physicochemical characteristics (Genot, Meynier, \& Riaublanc, 1999; Wanasundara \& Shahidi, 2005). Emulsifiers can influence the droplet charge, thickness, and permeability, all of which control the capacity of pro-oxidants, oxygen and free radicals to interact with the lipids in the droplets (Villiere, Viau, Bronnec, Moreau, \& Genot, 2005; Waraho et al., 2012). In fact, anionic emulsifiers may attract cationic transition metals, while cationic emulsifiers repulse them, thereby decreasing the rate of oxidation. (Silvestre, Chaiyasit, Brannan, McClements, \& Decker, 2000; Villiere et al., 2005). In addition, the interfacial region of an emulsion can form a physical (steric) barrier against the pro-oxidants dissolved in the aqueous phase. Thicker interfacial layers usually offer more protection, depending on the dimensions and composition of the emulsifier's head and tail group (Berton-Carabin, Ropers, \& Genot, 2014).

One of the best employed strategies to prevent oxidation in food systems is the addition of antioxidants (AOs) (Finley et al., 2011; Genot et al., 1999; Wanasundara \& Shahidi, 2005; Waraho, McClements, \& Decker, 2011). Nevertheless, we are still unable to predict how the intrinsic chemical structure of antioxidants affects their behavior in different food systems. In recent years, the lipophilization of hydrophilic molecules to synthesize antioxidants with a broad range of hydrophobicity has attracted interest (Lecomte, Giraldo, Laguerre, Baréa, \& Villeneuve, 2010; Lopez Giraldo et al., 2007). This is due to the possibility to fine-tune the hydrophobicity of a polyphenol by the grafting alkyl chains of different length and should help us to understand how antioxidant properties are affected by the hydrophobicity. To date, evidence supporting using different phenolipid series such as rosmarinates (Laguerre et al., 2010), hydroxytyrosyl esters (Almeida

\footnotetext{
* Corresponding author.

E-mail address: mpmartin@fc.up.pt (F. Paiva-Martins).
} 
et al., 2016; Medina, Lois, Alcántara, Lucas, \& Morales, 2009), caffeates (Costa, Losada-Barreiro, Paiva-Martins, Bravo-Díaz, \& Romsted, 2015) and chlorogenates (Meireles et al., 2019) in various oil-in-water emulsions has established that there is a nonlinear trend between hydrophobicity and antioxidant efficiency. Indeed, the dependence between the alkyl chain length and the antioxidant efficiency in emulsions usually follow a parabolic shape with a maximum for an intermediate alkyl length derivative (Laguerre et al., 2010, 2011). In attempting to understand this non-linear trend, the distribution of a series of AOs derived from hydroxytyrosol, caffeic acid, gallic acid, protocatechuic acid and chlorogenic acid in intact olive oil, soybean oil and corn-inwater emulsions was determined by employing a pseudophase kinetic model. We demonstrated that there is a positive correlation between the efficiency of $\mathrm{AOs}$ and the \% of $\mathrm{AO}$ at the interfacial region of emulsions (Almeida et al., 2016; Costa et al., 2015; Costa, LosadaBarreiro, Paiva-Martins, \& Bravo-Díaz, 2016, 2017; Losada-Barreiro, Bravo-Díaz, Paiva-Martins, \& Romsted, 2013; Losada-Barreiro, SánchezPaz, \& Bravo-Díaz, 2013; Meireles et al., 2019; Romsted \& Bravo-Díaz, 2013; Silva, Losada-Barreiro, Paiva-Martins, \& Bravo-Díaz, 2017). This fundamental result implies that the efficiency of AOs can be, in principle, increased by fine-tuning their interfacial concentrations.

Recently, the distribution of a homologous series of gallic acid (GA) derivatives of increasing lipophilicity was determined in stripped fish oil-in-water (Ferreira, Costa, Losada-Barreiro, Paiva-Martins, \& BravoDíaz, 2018) and results showed, again, that the antioxidant activity is positively correlated with the percentage of $\mathrm{AO}$ at the interface. Both butyl and octyl gallates were found to be at the highest concentration at the interfacial region and, therefore, the most suitable AOs to carry out protection of fish oil-in-water emulsions. Results also showed that for maximum effectiveness, butyl and octyl gallate should be used with the lowest emulsifier volume fraction necessary to physically stabilize emulsions because the increase in the emulsifier volume would result in a dilution of AOs at the interface region.

One of the hypotheses on which the application of the pseudophase model for the determination of the distribution of AOs is based is that its application is independent of the size of emulsion droplets. The experience of our research group in the study of the chemical reactivity in colloidal systems and colloids of association, together with the evidence from other researchers, makes us to believe that such hypothesis is correct, for two main reasons:

1) The good fit of the experimental data to the equations derived from the pseudophase model, and which assumes that the drop size does not affect the distribution results.

2) Chemical kinetics indicate that the rates of chemical reactions that take place in solution depend on the concentrations of the reactants and not on the reaction surface (Romsted \& Bravo-Díaz, 2013).

In fact, the transfer area affects the speed of reactions only in the case in which there is reactant transfer between phases (reactions in heterogeneous systems). This reagent transfer situation is not applicable to lipid oxidation reactions or inhibition reactions by antioxidants in emulsions. In fact, in emulsions, several domains can be distinguished in which the reaction can take place. However, these domains cannot be considered as true phases, since their limits are not clearly defined, as is the case for binary heterogeneous systems. Nevertheless, these domains or regions have different properties or characteristics and may be referred to as a "pseudophase" or region. In emulsions, a distinction can be made between the aqueous (W), oil (O) and interphase (I) regions.

In order to demonstrate experimentally the hypothesis that the distribution and effectiveness of antioxidants does not depend on the size of the emulsion droplets but on the concentration of reactants in the interphase region of the emulsion, the distribution and antioxidant efficiency of gallates was determined in nanoemulsions composed of fish oil, acidic water and Tween 80 as surfactant. All these experiments were carried out under identical conditions as those used in our previous work for the same compounds, the size of the drops being the only difference. Moreover, we also assessed the effect of the ratio water/oil used in the production of emulsions on gallates' antioxidant efficiency.

\section{Materials and methods}

\subsection{Materials}

Fish (FO) oil-in-water emulsions and nanoemulsions were prepared by employing commercial fish oil (generously provided by Biomega Natural Nutrients S.L., Coruña, Spain) stripped from their natural antioxidants (Lisete-Torres et al., 2012). Stripped oil was stored in the dark at $-20{ }^{\circ} \mathrm{C}$ to minimize its oxidation. The oil was composed (given as $\%$ total fatty acids) of $16 \%$ of eicosapentaenoic acid (EPA), $29 \%$ of docosahexaenoic acid (DHA), 23\% of saturated fatty acids, $25 \%$ of monounsaturated fatty acids and $52 \%$ of total $n-3$ polyunsaturated fatty acids (n-3 PUFA). The water employed in the preparation of all aqueous solutions was of Milli-Q grade (conductivity $<0.1 \mathrm{mS} \mathrm{cm}{ }^{-1}$ ). The citric acid and sodium citrate (Acros organics) employed in the preparation of buffer solutions were of the highest purity and used as received.

Gallic acid (GA), ethyl gallate (EG or C2), propyl gallate (PG or C3), butyl gallate (BG or C4), octyl gallate (OG or C8) and lauryl gallate (LG or C12) and Tween 80 were purchased from Sigma Aldrich and used as received. 4-Hexadecylbenzenediazonium tetrafluoroborate, 16$\mathrm{ArN}_{2} \mathrm{BF}_{4}$, was prepared from commercial 4-hexadecylaniline (Aldrich, 97\%) (Costa et al., 2015). Solutions of the coupling reagent $\mathrm{N}$-(1naphthyl)ethylenediamine (NED, Aldrich) were prepared in a 50:50 (v/ v) $\mathrm{BuOH}: \mathrm{EtOH}$ mixture to give $[\mathrm{NED}]=0.02 \mathrm{M}$.

\subsection{Cyclic voltammetry.}

The voltammetric working solutions were prepared, in the electrochemical cell using a glassy carbon working electrode and measured vs an $\mathrm{Ag} / \mathrm{AgCl}$ electrode, by diluting $0.1 \mathrm{~mL}$ of the stock solution $(10 \mathrm{mM}$ in ethanol) in $10 \mathrm{~mL}$ of aqueous phosphate buffer (0.1 M, pH 3.65). Stock solutions of each compound $(0.01 \mathrm{M})$ were prepared by dissolving an appropriate amount in ethanol. The voltammetric working solutions were prepared in the electrochemical cell by diluting $0.10 \mathrm{~mL}$ of the stock solution in $10 \mathrm{~mL}$ of supporting electrolyte in order to obtain a final concentration of $0.1 \mathrm{mM}$. The effect of emulsifier (at $2 \%$ of Tween $80)$ on the first anodic potential $\left(E_{\mathrm{pa}}\right)$ of the gallic acid and gallates was checked by carrying out auxiliary experiments both in the presence and absence of Tween 80 .

\subsection{Preparation of emulsions and nanoemulsions}

Fish oil-in-water $1: 9$ or $4: 6(\mathrm{v} / \mathrm{v})$ emulsions were prepared by mixing oil and an aqueous buffer (citric acid/citrate; $0.04 \mathrm{M}$; $\mathrm{pH} 3.65$ ) containing several surfactants (Tween 80) volume fractions, $\Phi_{\mathrm{I}}$ $\left(\Phi=\mathrm{V}_{\text {region }} / \mathrm{V}_{\text {total }}\right)$. When needed, $20 \mu \mathrm{L}$ of each antioxidant stock methanolic solution was added. The mixture was then homogenized at room temperature $\left(\mathrm{T}=20-22{ }^{\circ} \mathrm{C}\right)$ for 2 min with the aid of a highspeed rotor (Polytron PT $1600 \mathrm{E}$ ) at 20,000 rpm.

Nanoemulsions were prepared by placing the coarse emulsion in a high-pressure homogenizer (Nozzle Z5, Nano DeBEE, Bee International, USA). The device was covered with ice bags to avoid high temperatures caused by high-shear forces. A total of $50 \mathrm{~mL}$ of the coarse emulsion was subject to the homogenizer, using 3 cycles under the homogenization pressure of $17.2 \times 10^{7} \mathrm{~Pa}$.

\subsection{Physicochemical characterization of emulsions and nanoemulsions}

2.4.1. Average droplet size, polydispersity, and $\zeta$-potential measurements The droplet size and size distribution (polydispersity index, PDI) 
and $\zeta$-potential of the emulsified systems were measured by dynamic light scattering (DLS) (Zetasizer NanoZS laser diffractometer, Malvern Instruments Ltd., Worcestershire, UK) at $25{ }^{\circ} \mathrm{C}$. Intensity-weighted average droplet diameters were described as BZ-average and measurements were carried out in acrylic cuvettes (Labbox MAPS-F10-100) at $633 \mathrm{~nm}$ and at a fixed angle of $90^{\circ}$. To calculate the droplet size distribution, refractive indexes of 1.47 and 1.33 were employed for olive oil and water, respectively. Measurements were performed in triplicate. The size distribution was described by the polydispersity index (PDI) and the reported values are an average of 10 determinations. The PDI values obtained were $\leq 0.3$, indicating a narrow droplet size distribution. The $\zeta$-potential was determined by measuring the direction and velocity in which the droplets moved in an applied electric field by employing a particle electrophoresis instrument (Zetasizer Nanoseries Nano-ZS, Malvern Instruments, Worcestershire, UK). The Smoluchowski equation was used by the software to convert the electrophoretic mobility measurements into $\zeta$-potential values. For this purpose, freshly prepared emulsions were diluted to a droplet concentration of $\sim 0.001 \%(\mathrm{w} / \mathrm{v})$ with the same buffer solution employed in the preparation of the emulsion. Results are reported as the average of at least 5 measurements.

\subsubsection{Morphological characterization by Cryo-scanning electron microscopy (CSEM)}

The undiluted fish oil nanoemulsion containing a $\Phi_{\mathrm{I}}$ of $0.005,0.01$ and 0.02 was placed into carbon-coated grids and immersed in $\mathrm{N}_{2}$ for $30 \mathrm{~s}$. Next, it was transferred, under vacuum conditions, to a preparation chamber (Gatan Alto 2500, UK). Aliquots of the sample were fractured and sublimated at $-90{ }^{\circ} \mathrm{C}$ for $3 \mathrm{~min}$, and coated by ionic pulverization with $\mathrm{Au} / \mathrm{Pd}$ under $12 \mathrm{~mA}$ during $40 \mathrm{~s}$. CSEM images were recorded at $-150{ }^{\circ} \mathrm{C}$ using a scanning electron microscope (JEOL JSM 6301F/Oxford INCA Energy 350, Japan), with X-ray microanalyses and an observation system.

\subsection{Determining of the partition constants and distribution of gallates in intact fish oil-in-water nanoemulsions: Application of the pseudophase kinetic model}

Gallates distribute in a different extent between the oil, interfacial, or aqueous regions of emulsions depending upon their hydrophilic-lipophilic balance (HLB) and their distribution is defined by the partition constants between the oil-interfacial, $\mathrm{P}_{\mathrm{O}}^{\mathrm{I}}$, and aqueous-interfacial, $\mathrm{P}_{\mathrm{W}}^{\mathrm{I}}$, regions (Eqs. (1) and (2) and Fig. S1, Supporting Information).

$P_{O}^{I}=\frac{\left(A O_{I}\right)}{\left(A O_{O}\right)}$

$P_{W}^{I}=\frac{\left(A O_{I}\right)}{\left(A O_{W}\right)}$

being $\left(\mathrm{AO}_{\mathrm{I}}\right),\left(\mathrm{AO}_{\mathrm{O}}\right)$ and $\left(\mathrm{AO}_{\mathrm{W}}\right)$ the antioxidant concentration at the interfacial, oily and aqueous region, respectively (Supporting Information). The distribution of gallates was determined in the intact emulsions and nanoemulsions by employing a well-established kinetic method which exploits the rapid reaction between the gallates and the specifically synthetized chemical probe, 4-hexadecylbenzenediazonium $\left(16-\mathrm{ArN}_{2}{ }^{+}\right.$) ion. A derivatization method was used for monitoring the reaction of AOs with the chemical probe, $16-\mathrm{ArN}_{2}{ }^{+}$, and is based on the reaction of unreacted $16-\mathrm{ArN}_{2}{ }^{+}$with the coupling agent $\mathrm{N}$-(1-naphthyl) ethylenediamine, NED, to yield a stable azo dye whose absorbance can be measured spectrometrically. The probe is located at the interfacial region of emulsions because $16-\mathrm{ArN}_{2}{ }^{+}$is itself an ionic surfactant and because it is oil and water insoluble (Fig. S1, Supporting Information). Details of the method and the kinetic treatment are given elsewhere (Costa et al., 2015) and are briefly described in the Supporting Information.

\subsection{Antioxidant efficiency in fish oil emulsified systems.}

Antioxidant efficiency in emulsified systems was determined as in a previous work (Ferreira et al., 2018). Fish oil-in-water nanoemulsions (with a $\mathrm{O} / \mathrm{W}$ volume ratio of 1:9) and emulsions (with a volume $\mathrm{O} / \mathrm{W}$ ratio of $1: 9$ and $4: 6$ ) were prepared as above in the presence and absence (control samples) of gallates $\left([\mathrm{AO}]_{\mathrm{T}}=0.125 \mathrm{mM}\right.$ in total volume). Emulsified systems were thermostated at $\mathrm{T}=40{ }^{\circ} \mathrm{C}$ and vortexed for one minute every $12 \mathrm{~h}$. After each vortex, $20 \mu \mathrm{L}$ aliquot of the samples was used to determine the percentage of conjugated dienes (CDs) content (p/p) of the samples (Ferreira et al., 2018). Antioxidant efficiency was evaluated by determining the time necessary for the samples containing each compound to increase the conjugated diene content by $0.5 \%$. In order to evaluate the antioxidant efficiency of compounds in different emulsified systems at the same time, the relative increase in the oxidative stability was used (Eq. (3)):

Relative increase in the oxidative stability $=\left(t_{(\mathrm{AO})}-\mathrm{t}_{(\mathrm{C})}\right) / \mathrm{t}_{(\mathrm{C})}$

Being $t_{(\mathrm{AO})}$ and the $\mathrm{t}_{(\mathrm{C})}$ the time necessary for the samples containing each $\mathrm{AO}$ or the control to increase by $0.5 \%$ the conjugated diene content.

\subsection{Statistical analysis}

Kinetic experiments between the AOs and the chemical probe were run in triplicate for $2-3 t_{1 / 2}$. The observed constant rate $\left(k_{\text {obs }}\right)$ values were within $\pm 7-9 \%$ with typical correlation coefficients over 0.995 . All the cyclic voltammetry experiments were run at least in quadruplicate. All oxidation experiments were run in triplicate. SPSS 21.0 software was used for statistical analysis by one-way analysis of variance (ANOVA, with Tukey's HSD multiple comparison) with the level of significance set at $P<0.05$.

\section{Results and discussion}

\subsection{Physical characterization of emulsified systems.}

As expected, the droplet size of fish oil-in-water emulsions with oil water ratios of $1: 9$ and 4:6 obtained for an emulsifier volume fraction of 0.005 were much larger than those obtained for nanoemulsions with oil water ratios of $1: 9$. The average droplet size of the $1: 9$ and $4: 6$ oil water ratio coarse emulsions were $1282 \mathrm{~nm}$ and $4860 \mathrm{~nm}$, respectively (Table 1). The DLS plot for the coarse emulsions showed a bimodal

\section{Table 1}

Characteristics of the fish oil emulsions and nanoemulsions. $\Phi=\mathrm{V}_{\text {region }} / \mathrm{V}_{\text {total }}$; $\mathrm{O}$, oil region; I, interfacial region; $\mathrm{V}_{\text {total }}=10 \mathrm{~mL} ; \mathrm{d}=$ droplet diameter; $\mathrm{V}_{\text {droplet }}=\pi \mathrm{xd}^{3} / 6$, volume of one droplet; $\mathrm{S}_{\mathrm{d}}=\pi \mathrm{xd}^{2}$, droplet surface; $\mathrm{N}_{\mathrm{d}}=\mathrm{V}_{\text {total, oil }} / \mathrm{V}_{\text {droplet, }}$, total number of droplets $\left(\mathrm{V}_{\text {total, oil }}\right.$ is the total volume of oil per $10 \mathrm{~g}$ of emulsion); $S_{\text {total }}=6 \times \mathrm{V}_{\text {total, oil }} / \mathrm{d}$, surface of all droplets; $\mathrm{m}_{\mathrm{T} 80 \text {, }}$ droplet $=S_{\text {total }} \times \Gamma_{\infty}$, mass of surfactant required for saturation per $100 \mathrm{~g}$ of emulsion (estimated using a interfacial coverage at saturation of $\Gamma_{\infty}=2 \times 10^{-6}$ mol m${ }^{-2}$ (Berton-Carabin et al., 2014); $\mathrm{m}_{\mathrm{T} 80 \text {, excess }}=\mathrm{m}_{\mathrm{T} 80}-\mathrm{m}_{\mathrm{T} 80}$, droplet, excess (g) of Tween 80 that may remain in the aqueous phase.

\begin{tabular}{llllll}
\hline & \multicolumn{2}{l}{ Nanoemulsions } & \multicolumn{3}{c}{ Emulsions } \\
\hline$\Phi_{\mathrm{o}}$ & 1.0 & 1.0 & 1.0 & 1.0 & 4.0 \\
$10^{2} \Phi_{\mathrm{I}}$ & 0.5 & 1.0 & 2.0 & 0.5 & 0.5 \\
$\mathrm{~s}$-potential $(\mathrm{mV})$ & -18.1 & -14.2 & -13.5 & -22.6 & $\mathrm{nd}$ \\
$10^{6} \mathrm{~d}(\mathrm{~m})$ & 0.297 & 0.227 & 0.175 & 1.28 & 4.86 \\
$10^{12} \mathrm{~S}_{\text {droplet }}\left(\mathrm{m}^{2}\right)$ & 0.28 & 0.16 & 0.10 & 5.0 & 74.2 \\
$10^{20} \mathrm{~V}_{\text {droplet }}\left(\mathrm{m}^{3}\right)$ & 1.37 & 0.61 & 0.28 & 105 & 6007 \\
$10^{-12} \mathrm{~N}_{\mathrm{d}}$ & 73 & 163 & 357 & 9.5 & 0.67 \\
$\mathrm{~S}_{\text {total }}\left(\mathrm{m}^{2}\right)$ & 20.2 & 26.4 & 34.3 & 4.8 & 4.9 \\
$10^{2} \mathrm{~m}_{\text {T80 available }} / \mathrm{m}^{2}$ of Surface $(\mathrm{g})$ & 0.25 & 0.38 & 0.58 & 1.05 & 1.01 \\
$10^{2} \mathrm{~m}_{\text {T80, dropplet }}(\mathrm{g})$ & 0.53 & 0.69 & 0.90 & 0.12 & 0.13 \\
$10^{2} \mathrm{~m}_{\text {T80, excess }}(\mathrm{g})$ & -0.03 & 0.31 & 1.10 & 0.38 & 0.37
\end{tabular}


distribution with broad peaks with a PDI of almost 0.5 for the 1:9 emulsion and 0.8 for the $4: 6$ emulsion.

After passing the coarse 1:9 emulsion through the high-pressure homogenizer, the droplet size and the polydispersity index decreased notably, with a monomodal droplet size distribution. The average droplet size decreases upon increasing the emulsifier concentration (Fig. S2, Supplementary data), and a constant value lower than $150 \mathrm{~nm}$ was obtained above $\Phi_{\mathrm{I}}=0.03$. Probably, the higher amount of surfactant available at the oil-water interface is able to stabilize a higher interfacial area while reducing the interfacial tension, leading to lower droplet sizes. On the other hand, an increase in the amount of surfactant will increase the rate of coverage of any new droplet surfaces formed during homogenization (Qian \& McClements, 2011; Silva, Cerqueira, \& Vicente, 2015). Samples with PDI values of 0.3 and lower, as those obtained for the prepared nanoemulsions, indicate that they can be considered as monodisperse (McClements, 2015). To get further insights into the physical characteristics of the nanoemulsions and to analyze the potential presence of Tween 80 micelles (critical micelle concentration at $25{ }^{\circ} \mathrm{C}$ is $0.015 \mathrm{mM}$ ), we conducted a series of theoretical calculations to determine: the number of droplets in the system $\left(\mathrm{N}_{\mathrm{d}}\right)$; the total interfacial area of droplets $\left(\mathrm{S}_{\mathrm{d}}\right)$; the total mass of surfactant adsorbed at the interface of the droplets (assuming that the interface is saturated); and the mass of surfactant remaining in the aqueous phase after saturation (Table 1).

The $s$-values obtained for the prepared nanoemulsions showed a slight negative charge, as would be expected for emulsions stabilized with non-ionic surfactants such as Tween 80 . This negative charge increased upon increasing the emulsifier concentration, with values ranging from -18.2 to $-13.5 \mathrm{mV}$ (Table 1 and Fig. S2, Supplementary data). Absolute values are lower than $30 \mathrm{mV}$, suggesting that the main stabilization mechanism is due to steric stabilization. Interestingly, the $\varsigma$-potential of coarse emulsions with an oil water ratio of 1:9 were slightly higher $(-22.6 \mathrm{mV})$ than that found for nanoemulsions with the same oil water ratio and emulsifier concentration, suggesting that there is an increase in the s-potential upon increasing the droplet size (Table 1).

\subsection{Microscopic visualization of the nanoemulsion droplets.}

CSEM analysis allows the observation of colloidal systems in their natural form (Klang, Matsko, Valenta, \& Hofer, 2012). CSEM micrographs of fish oil nanoemulsions $(1: 9, \mathrm{O} / \mathrm{W})$ obtained with $\Phi_{\mathrm{I}}=0.005$, 0.01 and 0.02 showed the presence of clear spherical droplets with enough uniformity in the range of 233-175 $\mathrm{nm}$, revealing droplets of the oil phase surrounded by the aqueous phase (Fig. S3, Supplementary data). The presence of larger and smaller droplets in minor proportions, in particular at the lower emulsifier concentration can be described in different areas of the sample. This is common with nanoemulsions with a PDI of 0.19-0.24, particularly in undiluted samples (Saupe, Gordon, \& Rades, 2006). Droplet size data obtained by CSEM analysis were in accordance with the range of the droplet diameter obtained using the particle size analyzer (DLS).

\subsection{Effect of antioxidant chain length, emulsifier concentrations, $\mathrm{O} / W$} ratio on antioxidant distribution in fish oil nanoemulsions: Coarse emulsion and nanoemulsion comparison

The variations of $k_{\text {obs }}$ with $\Phi_{\mathrm{I}}$ for the reaction of $16 \mathrm{ArN}_{2}{ }^{+}$with AOs in intact 1:9 fish oil nanoemulsions can be observed in Fig. 1. In all runs, $k_{\text {obs }}$ values decrease 4.5 to 6 fold asymptotically, upon increasing the emulsifier volume fraction from $\Phi_{\mathrm{I}}=0.005$ to 0.04 . The solid lines in Fig. 1A, B and C are the theoretical curves obtained by fitting the $\left(k_{\mathrm{obs}}, \Phi_{\mathrm{I}}\right)$ pairs of data to Eq. (S3) (GA), Eq. (S1) (C2 and C4) and Eq. (S2) (C8 and C12), respectively. The excellent fits obtained, indicates that the assumptions of the pseudophase kinetic model mentioned in the introduction is fully justified (Costa et al., 2015). The slopes and intercepts of the linear plots of $1 / k_{\text {obs }}$ vs $\Phi_{\mathrm{I}}$ (straight lines in Fig. 1) were used to determine $\mathrm{P}_{\mathrm{W}}{ }^{\mathrm{I}}, \mathrm{P}_{\mathrm{O}}{ }^{\mathrm{I}}$ and the rate constant between the probe and $\mathrm{AO}$ at the interface region, $k_{\mathrm{I}}$. The corresponding values are shown in Table 2 (Costa et al., 2015). The $k_{\mathrm{I}}$ values obtained for gallic acid derivatives in nanoemulsions are similar, with an average value of $k_{\mathrm{I}}=0.31 \pm 0.08 \mathrm{M}^{-1} \mathrm{~s}^{-1}$. This is in line with the negligible effect of chain length on the reactivity of GA in homogeneous solution with the DPPH radical and with the slight variations in the first anodic peak potential $\left(E_{\mathrm{pa}}\right)$ determined by cyclic voltammetry (Table 2). The small differences in the $k_{\mathrm{I}}$ values suggest that the AOs phenolic moieties are located in similar reaction environments.

Knowing the partition constants, $P_{\mathrm{W}}{ }^{\mathrm{I}}$ and $P_{\mathrm{O}}{ }^{\mathrm{I}}$, the AO percentage in the interfacial region, $\% \mathrm{AO}_{\mathrm{I}}$, oil, $\% \mathrm{AO}_{\mathrm{O}}$, and aqueous, $\% \mathrm{AO}_{\mathrm{W}}$, regions were determined (Fig. 1D, 1E and 1F). Fig. 1D shows that at $\Phi_{\mathrm{I}}=0.005$, more than $50 \%$ of GA derivatives are located in the interfacial region and that $\% \mathrm{AO}_{\mathrm{I}}$ follows the order \%GA $<\% \mathrm{C} 2<$ $\% \mathrm{C} 4<\% \mathrm{C} 12<\%$ C8. This percentage increases upon increasing $\Phi_{\mathrm{I}}$ so that at $\Phi_{\mathrm{I}}=0.04$, more than $90 \%$ of all AOs are located in the interfacial region and at $\Phi_{\mathrm{I}}$ greater than 0.02 , the $\% \mathrm{AO}_{\mathrm{I}}$ becomes almost independent of the alkyl chain length (Fig. 1D). These results are in accordance with those previously reported by us for emulsions with a similar composition (Ferreira et al., 2018). In fact, very similar $P_{\mathrm{W}}{ }^{\mathrm{I}}$ and $P_{\mathrm{O}}^{\mathrm{I}}$ (differences lower than $6 \%$ ) were obtained for the distribution of these compounds in fish oil-in-water emulsions, (Table 2) showing that distribution of gallates is not affected by the droplet size (Fig. 2).

By calculating the interfacial molarities of $\mathrm{AOs},\left(\mathrm{AO}_{\mathrm{I}}\right)$, a dilution of the AOs at the interfacial region as a result of the increase in $\Phi_{\mathrm{I}}$, is observed, as shown in Fig. 3. Thus, for any $\mathrm{AO}$, the $\left(\mathrm{AO}_{\mathrm{I}}\right)$ decreases asymptotically 6 to 7 fold upon increasing $\Phi_{\mathrm{I}}$ from $\Phi_{\mathrm{I}}=0.005$ to $\Phi_{\mathrm{I}}=0.04$. Therefore, for any $\mathrm{AO}$ at fixed oil water ratio, the increase in the AO percentage at the interfacial region does not compensate the increase in the interfacial volume and a dilution takes place, i.e., $\left(\mathrm{AO}_{\mathrm{I}}\right)$ decreases with increasing $\Phi_{\mathrm{I}}$. Thus, at any given stoichiometric (or added) AO concentration, $[\mathrm{AO}]_{\mathrm{T}}$, the effective $(\mathrm{AO})_{\mathrm{I}}$ depends on both $\% \mathrm{AO}_{\mathrm{I}}$ and $\Phi_{\mathrm{I}}$. Fig. $3 \mathrm{~B}$ shows that at any $\Phi_{\mathrm{I}}$, a parabolic-like variation of the $(\mathrm{AO})_{\mathrm{I}}$ is observed, increasing up to a limit, upon increasing the hydrophobicity of the AOs, after which (AO) I decreases. However, the variations in (AO) $)_{I}$ with HLB are less pronounced at high $\Phi_{I}$ values because most of the AOs are already located in the interfacial region (Fig. 3B).

Results show that the $\left(\mathrm{AO}_{\mathrm{I}}\right)$ of any of the GA alkyl derivatives are much higher $\left(\sim 110-190\right.$ fold at $\Phi_{\mathrm{I}}=0.005$ and $\sim 55-95$ fold at $\left.\Phi_{\mathrm{I}}=0.01\right)$ than the stoichiometric concentration of the AOs (0.125 mM). The highest local AO concentration was obtained when employing the lower surfactant concentration needed to stabilize kinetically the emulsions. The distribution results clearly show that, the main parameter controlling the distribution of gallates in emulsified systems is the emulsifier concentration, and that changes in the HLB of the AOs may be less effective than the changes in $\Phi_{\mathrm{I}}$. For example, the interfacial molarity for the $\mathrm{C} 8$ derivative decreases 8 -fold with increasing $\Phi_{\mathrm{I}}$ from 0.005 to 0.02 , but, at constant $\Phi_{\mathrm{I}},\left(\mathrm{AO}_{\mathrm{I}}\right)$ only increases 1.1 to 2.5 fold on going from GA to C8 (Fig. $3 \mathrm{~B}$ ). On the other hand, the $\%$ of $\mathrm{AO}$ remaining in the aqueous phase may have an important negative effect on the overall stability of the emulsified systems and a small increase in the $\left(\mathrm{AO}_{\mathrm{W}}\right)$ may significantly decrease the stability of these systems.

3.4. Effect of antioxidant chain length, emulsifier concentrations and $O / W$ ratio on antioxidant efficiency in fish oil nanoemulsions: Coarse emulsion and nanoemulsion comparison. Correlation distribution-AO efficiency in emulsions and nanoemulsions

Since the rate of a chemical reactions depends on the concentration of reactants at the reaction site, the antioxidant efficiency of compounds should depend on the AO interfacial concentration. Therefore, we hypothesized in previous reports, that there should be a direct 

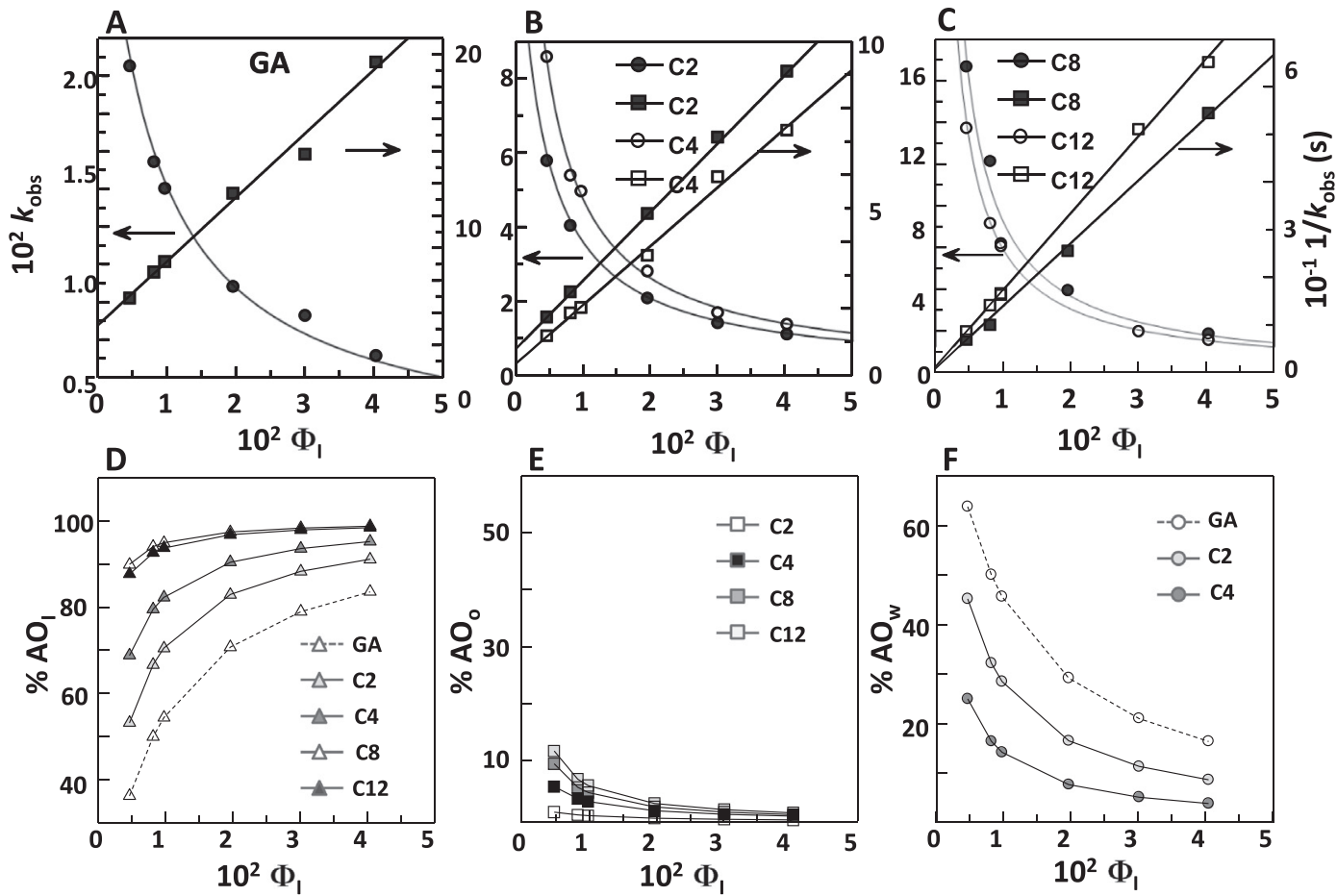

Fig. 1. Changes in the observed rate constant $\left(k_{\mathrm{obs}}\right)$ and $1 / k_{\mathrm{obs}}$ with $\Phi_{\mathrm{I}}$ for $(\mathrm{A}) \mathrm{GA}(\mathrm{B}) \mathrm{C} 2$ and $\mathrm{C} 4$ and (C) $\mathrm{C} 8$ and C12. $[\mathrm{AO}]=3.2 \times 10^{-3} \mathrm{M}, \mathrm{T}=25^{\circ} \mathrm{C}$. The solid lines are the theoretical curves obtained by fitting the ( $\left.k_{\mathrm{obs}}, \Phi_{\mathrm{I}}\right)$ pairs of data to Eq. (S3), Supporting Information (GA), Eq. (S1), Supporting Information (C2 and C4 derivatives) and Eq. (S2), Supporting Information (C8 and C12) and their reciprocals. Percentages of GA and GA alkyl esters in the interfacial (D), oil (E) and aqueous (F) regions of 1:9 $(\mathrm{O} / \mathrm{W})$ fish oil nanoemulsions.

Table 2

AO percentages in water $\left(\% \mathrm{AO}_{\mathrm{W}}\right)$ and $P_{\mathrm{W}}{ }^{\mathrm{O}}$ values in binary fish oil-water systems, in the absence of emulsifier, $P_{\mathrm{O}}{ }^{\mathrm{I}}$ and $P_{\mathrm{W}}{ }^{\mathrm{I}}$ values and the rate constant in the interfacial region, $k_{\mathrm{I}}$ in fish oil emulsions and in fish oil nanoemulsions. $\mathrm{EC}_{50}$ (mole $\mathrm{AO} /$ mole $\mathrm{DPPH}^{\bullet}$ ) values obtained at different reaction times. $E_{\mathrm{pa}}$, first anodic peak potentials. * Data extracted from Ferreira et al., 2018.

\begin{tabular}{|c|c|c|c|c|c|c|}
\hline & & GA & $\mathrm{C}_{2}$ & $\mathrm{C}_{4}$ & $\mathrm{C}_{8}$ & $\mathrm{C}_{12}$ \\
\hline \multirow[t]{2}{*}{ Binary System* } & $\% \mathrm{AO}_{\mathrm{W}}$ & $96.5 \pm 2.1$ & $82.1 \pm 0.5$ & $40.4 \pm 1.1$ & $\approx 0$ & $\approx 0$ \\
\hline & $P_{\mathrm{W}}{ }^{\mathrm{O}}$ & $0.05 \pm 0.03$ & $0.330 \pm 0.012$ & $2.21 \pm 0.10$ & - & - \\
\hline \multirow[t]{3}{*}{ Emulsion $\mathrm{O} / \mathrm{W}^{*}$} & $P_{\mathrm{W}}^{\mathrm{I}}$ & $118 \pm 19$ & $233 \pm 32$ & $559 \pm 127$ & - & - \\
\hline & $P_{\mathrm{O}}^{\mathrm{I}}$ & - & $706 \pm 56$ & $253 \pm 56$ & $183 \pm 26$ & $142 \pm 37$ \\
\hline & $10^{2} k_{\mathrm{I}}\left(\mathrm{M}^{-1} \mathrm{~s}^{-1}\right)$ & $0.082 \pm 0.003$ & $0.106 \pm 0.005$ & $0.162 \pm 0.019$ & $0.148 \pm 0.009$ & $0.133 \pm 0.005$ \\
\hline \multirow[t]{3}{*}{ Nanoemulsion $\mathrm{O} / \mathrm{W}$} & $P_{\mathrm{W}}^{\mathrm{I}}$ & $109 \pm 19$ & $247 \pm 32$ & $578 \pm 127$ & - & - \\
\hline & $P_{\mathrm{O}}^{\mathrm{I}}$ & - & $750 \pm 27$ & $261 \pm 21$ & $189 \pm 39$ & $151 \pm 24$ \\
\hline & $10^{2} k_{\mathrm{I}}\left(\mathrm{M}^{-1} \mathrm{~s}^{-1}\right)$ & $0.13 \pm 0.003$ & $0.24 \pm 0.005$ & $0.30 \pm 0.019$ & $0.39 \pm 0.009$ & $0.31 \pm 0.005$ \\
\hline \multirow[t]{2}{*}{$\mathrm{EC}_{50}{ }^{\mathrm{a}} *$} & $5 \mathrm{~min}$ & $0.105 \pm 0.002$ & $0.118 \pm 0.003$ & $0.113 \pm 0.002$ & $0.103 \pm 0.003$ & $0.097 \pm 0.004$ \\
\hline & $60 \mathrm{~min}$ & $0.087 \pm 0.001$ & $0.105 \pm 0.002$ & $0.098 \pm 0.003$ & $0.094 \pm 0.002$ & $0.085 \pm 0.003$ \\
\hline \multirow[t]{2}{*}{$E_{\mathrm{pa}}(\mathrm{mV})$} & $0 \%$ Tween 80 & 0.348 & 0.361 & 0.369 & 0.353 & 0.360 \\
\hline & $2 \%$ Tween 80 & 0.355 & nd & nd & 0.367 & 0.370 \\
\hline
\end{tabular}

a Data are presented as means \pm standard deviation $(n=4)$.

relationship between $\left(\mathrm{AO}_{\mathrm{I}}\right)$ and their efficiency in emulsions (Almeida et al., 2016; Costa etal., 2016, 2017; Losada-Barreiro et al., 2013a; Silva et al., 2017). Moreover, since the distribution of each compound has not been affected by the droplet size, a similar ranking of antioxidant activity should be obtained for the series of gallates when tested in fish oil-in-water emulsions and nanoemulsions.

To confirm our hypothesis, we determined the AO efficiencies in the same fish oil nanoemulsions employed in the distribution experiments. Prior to determining the AOs efficiency, we wanted to make sure that the alkyl chain length has a negligible effect on the AOs reactivity. Therefore, in a previous work (Ferreira et al., 2018), the antiradical efficiency of compounds in bulk solution by employing the $\mathrm{DPPH}^{-}$assay was investigated (Table 2). In accordance with results previously found for some gallates and for other antioxidants (Almeida et al., 2016; Costa et al., 2015; Losada-Barreiro et al., 2013a; Silva et al., 2017), EC $_{50}$ values were essentially independent of the length of the alkyl chain.
Correlation between antioxidant profile and redox properties of low molecular weight antioxidants is also well established (Chevion, Chevion, Chock, \& Beecher, 1999). Results presented in Table 2 show that values for the first anodic potential are independent of the length of the alkyl moiety and do not change, even in the presence of the emulsifier.

Fig. 3A shows the effect of the alkyl chain of AOs on the oxidative stability of nanoemulsions, measured as the time needed to increase by $0.5 \%$ the initial content of CDs. These results show that the efficiency of the AOs improves significantly $(p<0.05)$ upon lipophilization of GA in nanoemulsions prepared with $\Phi_{\mathrm{I}}=0.005$. The order of relative efficiency at all $\Phi_{\mathrm{I}}$ tested is $\mathrm{C} 8>\mathrm{C} 12>\mathrm{C} 4>\mathrm{C} 2>\mathrm{GA} \approx$ control, i.e., there is no correlation with their HLB. This increase in the activity of a series of compounds bearing the same reactive moiety, with their HLB up to a maximum, after which the activity decreases, is known as the "cut-off" effect (Losada-Barreiro et al., 2013a). Since we employed 


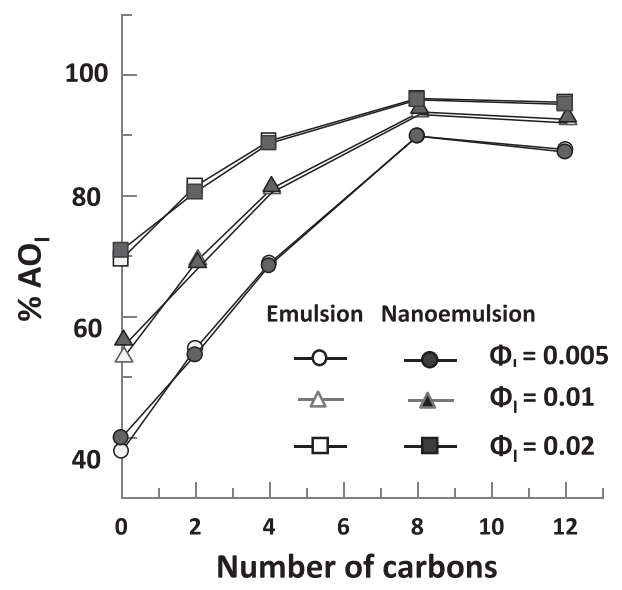

Fig. 2. Comparison of the $\% \mathrm{AO}_{\mathrm{I}}$ in $1: 9, \mathrm{O} / \mathrm{W}$ emulsions and nanoemulsions at $\Phi_{\mathrm{I}}=0.005,0.01$ and 0.02 .

AOs with similar reactivity against free radicals in solution, results show that this effect is a consequence of the differential partitioning of the reactants (Almeida et al., 2016; Costa et al., 2016, 2017; LosadaBarreiro et al., 2013a; Silva et al., 2017). Thus, we hypothesize that the relative order in AO efficiency should be mainly attributable to the differential concentrations of the AOs in the interfacial region of the emulsions.

In order to prove this hypothesis, we plotted the variation in $\left(\mathrm{AO}_{\mathrm{I}}\right)$ and the time to reach $0.5 \% \mathrm{CD}$ (Fig. 3B). We could observe that the variation of the $\mathrm{AO}$ efficiency with the chain length matches that of the interfacial concentration, confirming that there is a positive correlation between the AO efficiency and its interfacial concentration, independent of the $\Phi_{I}$ used in the preparation of the nanoemulsions and the droplet size. We can also observe that the ranking of effectiveness for gallates in nanoemulsions is the same as that obtained in our previous work with emulsions, in accordance with the similar distribution found in both these emulsifier systems. Therefore, our results support a model in which the antioxidant efficiency correlates with the antioxidant interfacial concentration, suggesting that we can also modulate AO efficiency in nanoemulsions by modifying the HLB of the AOs. We also observed the dilution effect on the antioxidant activity of compounds. Results showed that the highest local AO concentration is obtained when employing the lower surfactant concentration needed to stabilize kinetically the emulsions and, therefore, the oxidative stability of nanoemulsions decreases with the increase in the $\Phi_{I}$ (Fig. 3).

It is interesting to note that nanoemulsions prepared with $\Phi_{\mathrm{I}}=0.02$ in the absence of AOs, with the lowerst droplet size and, as consequence with the highest total interfacial surface, showed more oxidative stability than the ones prepared with less quantity of emulsifier (Fig. 3). Taking in account the values found in table 1 , results suggest that for the lowest surfactant concentration employed in this case, might not be sufficient surfactant to completely saturate the interfacial region. Consequently, no, or very few micelles are present in nanoemulsions containing only $\Phi_{\mathrm{I}}=0.005$. On the other hand, the amount of emulsifier used to stabilize nanoemulsions containing $\Phi_{\mathrm{I}}=0.01$ and, even in a greater extent, nanoemulsions containing $\Phi_{\mathrm{I}}=0.02$ is much higher than those required to only recover the droplet surface (Table 1). This suggests that, not only the presence of Tween 80 micelles, but also that the packing of surfactant molecules at the oil-water interface is tighter. This better coverage of droplets, may shield the lipidic droplets to the pro-oxidant species dissolved in the aqueous phase, increasing the stability of the system. Non-ionic surfactant micelles can come very near the drops, owing to the absence of electrical repulsion, as shown by the very low $s$-potential. Surfactants and oil molecules exchange very fast. The exchange of surfactants between micelles and the aqueous solution taking place in around $10^{-6} \mathrm{~s}$, and the mean lifetime of a micelle is around $10^{-3} \mathrm{~s}$ (Romsted, 2012). Thus, both micelles and nanoemulsion drops can acquire excess of surfactant by adsorption of the surfactant at the oil-water interface from micelles that coalesce or dissociate nearby the droplets, allowing surfactant molecules to be adsorbed (Gruner et al., 2016; Malassagne-Bulgarelli \& McGrath, 2009). Micelles in the aqueous phase, even if there are dynamic structures with very fast exchange rates, may also trap molecules such as AOs, hydroperoxides and other pro-oxidants (Berton-Carabin et al., 2014; Kargar, Spyropoulos, \& Norton, 2011). Results demosnstrate, as we initially assumed, that the rates of chemical reactions that take place during oxidation depend on the concentrations of the reactants and not on the reaction surface. In fact, with the increase of $\Phi_{\mathrm{I}}$, both AOs and radicals become diluted in the interfacial region.

The oxidative stability of emulsions can vary widely with small differences in the experimental conditions and with small differences in the oil used in the experiment. In order to confirm the results obtained in this work and our previous results with emulsions, an experiment assessing the antioxidant capacity of compounds in 1:9 fish oil-in water $\left(\Phi_{\mathrm{I}}=0.005\right)$ emulsions (zero passes) and nanoemulsions ( 3 passes in the homogenizer) at the same time (Fig. 4) were performed. We also ran
A

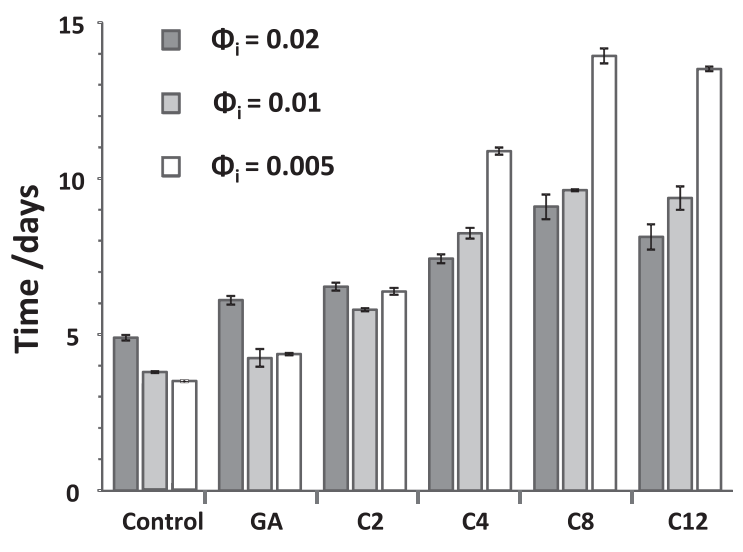

B

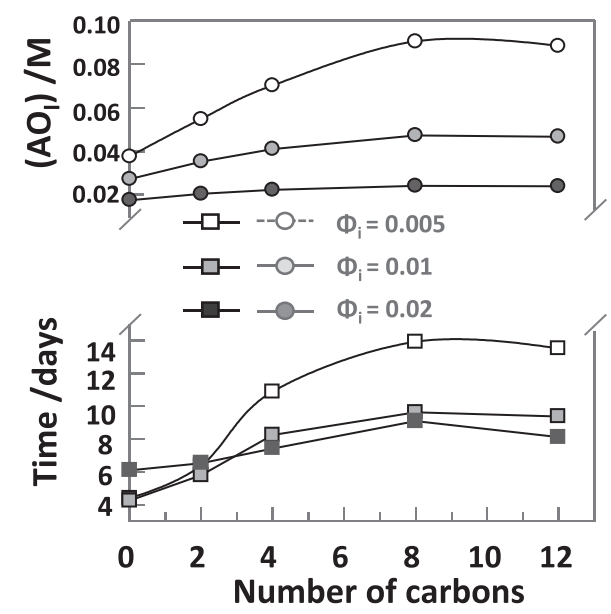

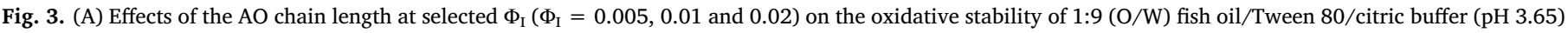

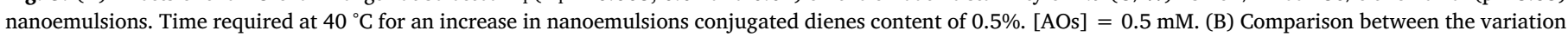

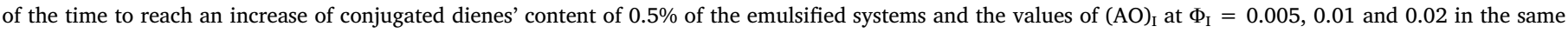
systems. 
A

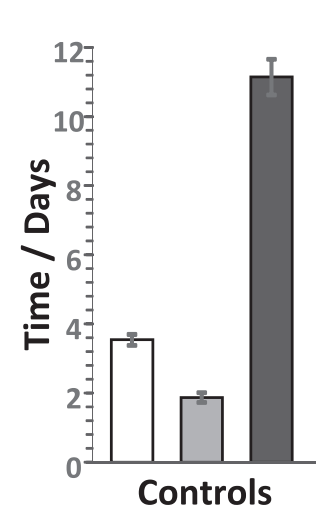

B

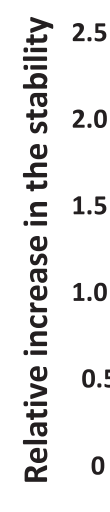

\section{.}

C

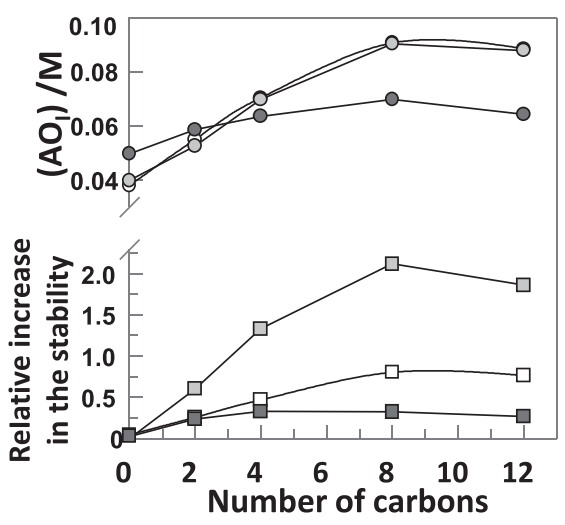

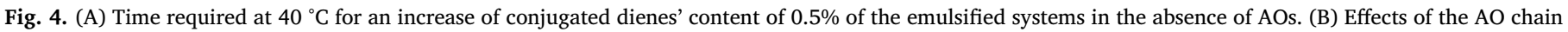

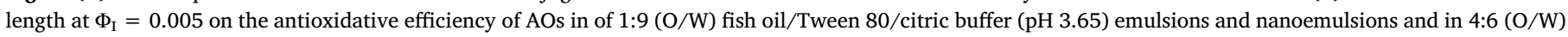

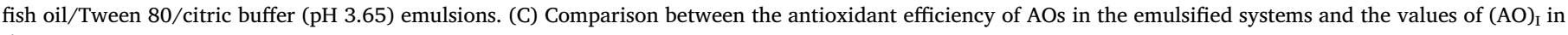
the same systems at $\Phi_{\mathrm{I}}=0.005$.

in the same experiment, emulsions with an oil water ratio of 4:6 but with $\Phi_{\mathrm{I}}=0.005$, in order to assess the effect of a different oil water ratio on the antioxidant efficiency of compounds.

In the absence of AOs, the three emulsified systems showed different oxidative stability, being the emulsions containing an oil water ratio of 4:6 the more stable ones. This probably results from the more surfaceactive radicals diffusing better into the interfacial region than into the oil region and therefore, the inner part of the droplets becoming inaccessible to direct interaction with the free radicals. This also implies a lower ratio of oxidizable fatty acid chains located near the interface to fatty acid chains embedded in the hydrophobic core of the droplets. On the other hand, a lower fraction of aqueous phase may decrease the amount of hydrosoluble pro-oxidants. This result is in line with those obtained by other authors (Kargar et al., 2011; Osborn \& Akoh, 2004; Sun \& Gunasekaran, 2009). Important to note is that nanoemulsions and emulsions with the same composition showed different stability, with the nanoemulsion system showing more stability than the coarse emulsion, even when the emulsified system had the highest total interfacial surface (Table 1). This confirms that oxidation reactions do not depend on the extension of the interfacial surface. In fact, no positive relationship between oxidative instability and extension of the interfacial surface was found. The lower oxidative stability of the 1:9 emulsions when compared with the 1:9 nanoemulsions may be related with their physical stability. Nanoemulsions were visually stable for months (more than 6 months) at $4{ }^{\circ} \mathrm{C}$. On the other hand, emulsions with the same composition, showed some creaming after a few days. Nevertheless, it is important to point out that no phase separation was observed in these emulsions even after 4 weeks at $4{ }^{\circ} \mathrm{C}$. Even at $40{ }^{\circ} \mathrm{C}$, phase separation was only observed when samples were oxidized, probably due not only to the oxidation products formed but also to the emulsifier oxidation (Kerwin, 2008). Hydroperoxides are more surfaceactive than their non-peroxidized counterparts (Nuchi, Hernandez, McClements, \& Decker, 2002). This may lead to reorganization and desorption of some emulsifiers from the interface and contribute to physical destabilization of the system (Genot, Berton, \& Ropers, 2013). Therefore, during the stability test, oxidized fatty acids and oxidized emulsifier molecules may come into contact with other emulsifier molecules and lipids from other droplets more easily if creaming phenomena occurs. Moreover, creaming brings droplets closer to ambient air.

Since the initial stability of the emulsified systems was different (Fig. 4), and in order to evaluate the antioxidant efficiency of compounds, instead of the time for the samples to get a $0.5 \%$ conjugated diene content, the increase in the oxidative stability ratio was used. Once more, a correlation between the AO efficiency and its interfacial concentration was found (Fig. 4). As expected, the antioxidant efficiency of the more lipophilic compounds (C8 and C12) in the 4:6 oil-inwater emulsions decreased when compared to the efficiency of the more hydrophilic compounds ( $C 2$ and C4). The increase in the $\Phi_{O}$ caused a shift of the compounds in the interphase region to the oil region. Consequently, in this emulsified system only small differences between the efficiency of all gallates could be observed (Fig. 4). On the other hand, in nanoemulsions and emulsions with the same composition (1:9), the same ranking of antioxidant activity for gallates was observed. However, the antioxidant protection given by the compounds was more important in the case of the more oxidative unstable emulsified system (Fig. 4), namely the (1:9) emulsions.

\section{Conclusions}

The distribution of a series of gallates of increasing lipophilicity has been determined by the pseudophase model, both in coarse fish oil in water emulsions and in nanoemulsions. One of the hypotheses on which the pseudophase model is based is that its application is independent of the size of the emulsion droplets. In agreement with our hypothesis, the results show that the smaller droplet size found in nanoemulsions does not affect partition constants of gallic acid and its esters. The antioxidant efficiency of GA and its gallates in the emulsified systems used was always positively correlated with the concentration of antioxidant at the interface region in all the emulsified systems tested. Beside the droplet size being smaller, the oxidative stability of nanoemulsions was higher than the oxidative stability of the coarse emulsion used in their preparation. This indicates that the rates of chemical reactions during oxidation depend on the concentrations of the reactants and not on the reaction surface, as we initially assume. The increase in the oil/water ratio increased the overall oxidative stability of emulsions. However, it decreased the antioxidant efficiency of the more lipophilic derivatives when compared with the more hydrophilic ones. Thus, the increase in the oil phase volume, decreased the interfacial concentration of this more lipophilic antioxidant.

The concentration of surfactant employed to stabilize kinetically the emulsions also plays a crucial role and should be kept as low as possible (Fig. 3) when AOs are used to control their stability. An increase in the surfactant volume fraction promotes incorporation of AOs into the interfacial region but at the same time increases the interfacial volume, leading to an effective dilution of $\mathrm{AO}_{\mathrm{S}}$ in that region (Fig. 3) decreasing 
their efficiency.

The results obtained highlight the importance of determining the distributions of AOs in emulsified systems, since they can be used to predict the ability of AOs to inhibit lipid oxidation. Furthermore, they allow a deeper knowledge of the effects of a number of experimental parameters on the antioxidant efficiency in these systems. Results provide fundamental information on the factors that control antioxidant distribution, which may be valuable in the preparation of new functional, lipid-based foods such as parenteral emulsified systems, where increasing amounts of fish oils are being incorporated in their formulations.

\section{Declaration of Competing Interest}

The authors declare that they have no known competing financial interests or personal relationships that could have appeared to influence the work reported in this paper.

\section{Acknowledgement}

We would like to thank Prof. Larry Romsted (Rutgers, The State University of New Jersey, USA) for encouragement and extremely fruitful discussions. Financial support of the following institutions is acknowledged: Red de Uso Sostenible de los Recursos Naturales y Agroalimentarios (REDUSO, Xunta de Galicia, Grant number ED431D 2017/18), FEDER(COMPETE); FCT - Fundação para a Ciência e a Tecnologia (UID/QUI/50006/2013 - POCI-01-0145-FEDER-007265); University of Vigo and University of Porto. S. L-B thanks Xunta de Galicia for a postdoctoral grant (POS-B/2016/012) and University of Vigo for "Plan de Retención de Talento 2018" grant. MC thanks FCT for the doctoral grant (SFRH/BD/100889/2014).

\section{Appendix A. Supplementary data}

Supplementary data to this article can be found online at https:// doi.org/10.1016/j.foodchem.2019.125716.

\section{References}

Almeida, J., Losada-Barreiro, S., Costa, M., Paiva-Martins, F., Bravo-Díaz, C., \& Romsted, L. S. (2016). Interfacial concentrations of hydroxytyrosol and its lipophilic esters in intact olive oil-in-water emulsions: Effects of antioxidant hydrophobicity, surfactant concentration, and the oil-to-water ratio on the oxidative stability of the emulsions. Journal of Agricultural and Food Chemistry, 64(25), https://doi.org/10.1021/acs.jafc. 6b01468.

Berton-Carabin, C. C., Ropers, M.-H., \& Genot, C. (2014). Lipid oxidation in oil-in-water emulsions: Involvement of the interfacial layer. Comprehensive Reviews in Food Science and Food Safety, 13(5), 945-977. https://doi.org/10.1111/1541-4337.12097.

Chevion, S., Chevion, M., Chock, P. B., \& Beecher, G. R. (1999). Antioxidant capacity of edible plants: Extraction protocol and direct evaluation by cyclic voltammetry. Journal of Medicinal Food, 2(1), 1-10. https://doi.org/10.1089/jmf.1999.2.1.

Costa, M., Losada-Barreiro, S., Paiva-Martins, F., \& Bravo-Díaz, C. (2016). Optimizing the efficiency of antioxidants in emulsions by lipophilization: Tuning interfacial concentrations. RCS Advances, 6(94), https://doi.org/10.1039/c6ra18282h.

Costa, M., Losada-Barreiro, S., Paiva-Martins, F., \& Bravo-Díaz, C. (2017). Physical evidence that the variations in the efficiency of homologous series of antioxidants in emulsions are a result of differences in their distribution. Journal of the Science of Food and Agriculture, 97(2), https://doi.org/10.1002/jsfa.7765.

Costa, M., Losada-Barreiro, S., Paiva-Martins, F., Bravo-Díaz, C., \& Romsted, L. S. (2015). A direct correlation between the antioxidant efficiencies of caffeic acid and its alkyl esters and their concentrations in the interfacial region of olive oil emulsions. The pseudophase model interpretation of the "cut-off" effect. Food Chemistry, 175. https://doi.org/10.1016/j.foodchem.2014.10.016.

Ferreira, I., Costa, M., Losada-Barreiro, S., Paiva-Martins, F., \& Bravo-Díaz, C. (2018). Modulating the interfacial concentration of gallates to improve the oxidative stability of fish oil-in-water emulsions. Food Research International, 112. https://doi.org/10. 1016/j.foodres.2018.06.007.

Finley, J. W., Kong, A.-N., Hintze, K. J., Jeffery, E. H., Ji, L. L., \& Lei, X. G. (2011). Antioxidants in foods: State of the science important to the food industry. Journal of Agricultural and Food Chemistry, 59(13), 6837-6846. https://doi.org/10.1021/ jf2013875.

Genot, C., Berton, C., \& Ropers, M.-H. (2013). The role of the interfacial layer and emulsifying proteins in the oxidation in oil-in-water emulsions. Lipid Oxidation, 177-210. https://doi.org/10.1016/B978-0-9830791-6-3.50008-4.
Genot, C., Meynier, A., \& Riaublanc, A. (1999). Lipid oxidation in emulsions. In Afaf Kalman-Eldin (Ed.). Lipid Oxidation Pathways. USA: AOC Press.

Gruner, P., Riechers, B., Semin, B., Lim, J., Johnston, A., Short, K., \& Baret, J.-C. (2016) Controlling molecular transport in minimal emulsions. Nature Communications, 7(1), 10392. https://doi.org/10.1038/ncomms10392.

Kargar, M., Spyropoulos, F., \& Norton, I. T. (2011). The effect of interfacial microstructure on the lipid oxidation stability of oil-in-water emulsions. Journal of Colloid and Interface Science, 357(2), 527-533. https://doi.org/10.1016/j.jcis.2011.02.019.

Kerwin, B. A. (2008). Polysorbates 20 and 80 used in the formulation of protein biotherapeutics: Structure and degradation pathways. Journal of Pharmaceutical Sciences, 97(8), 2924-2935. https://doi.org/10.1002/jps.21190.

Klang, V., Matsko, N. B., Valenta, C., \& Hofer, F. (2012). Electron microscopy of nanoemulsions: An essential tool for characterisation and stability assessment. Micron, 43(2-3), 85-103. https://doi.org/10.1016/j.micron.2011.07.014.

Laguerre, M., Chen, B., Lecomte, J., Villeneuve, P., McClements, D. J., \& Decker, E. A. (2011). Antioxidant properties of chlorogenic acid and its alkyl esters in stripped corn oil in combination with phospholipids and/or water. Journal of Agricultural and Food Chemistry, 59(18), 10361-10366. https://doi.org/10.1021/jf2026742.

Laguerre, M., López Giraldo, L. J., Lecomte, J., Figueroa-Espinoza, M.-C., Baréa, B., Weiss, J., ... Villeneuve, P. (2010). Relationship between hydrophobicity and antioxidant ability of "phenolipids" in emulsion: A parabolic effect of the chain length of rosmarinate esters. Journal of Agricultural and Food Chemistry, 58(5), 2869-2876. https://doi.org/10.1021/jf904119v.

Lecomte, J., Giraldo, L. J. L., Laguerre, M., Baréa, B., \& Villeneuve, P. (2010). Synthesis, characterization and free radical scavenging properties of rosmarinic acid fatty esters. Journal of the American Oil Chemists' Society, 87(6), 615-620. https://doi.org/10. 1007/s11746-010-1543-8.

Lisete-Torres, P., Losada-Barreiro, S., Albuquerque, H., Sánchez-Paz, V., Paiva-Martins, F., \& Bravo-Díaz, C. (2012). Distribution of hydroxytyrosol and hydroxytyrosol acetate in olive oil emulsions and their antioxidant efficiency. Journal of Agricultural and Food Chemistry, 60(29), 7318-7325. https://doi.org/10.1021/jf301998s.

Lopez Giraldo, L. J., Laguerre, M., Lecomte, J., Figueroa-Espinoza, M.-C., Pina, M., \& Villeneuve, P. (2007). Lipophilisation de composés phénoliques par voie enzymatique et propriétés antioxydantes des molécules lipophilisées. OCL. Oléagineux Corps Gras Lipides, 14(1), 51-59. https://doi.org/10.1684/ocl.2007.0100.

Losada-Barreiro, S., Bravo-Díaz, C., Paiva-Martins, F., \& Romsted, L. S. (2013). Maxima in antioxidant distributions and efficiencies with increasing hydrophobicity of gallic acid and its alkyl esters. The pseudophase model interpretation of the "cutoff effect". Journal of Agricultural and Food Chemistry, 61(26), 6533-6543. https://doi.org/10. 1021/jf400981x.

Losada-Barreiro, S., Sánchez-Paz, V., \& Bravo-Díaz, C. (2013). Effects of emulsifier hydrophile-lipophile balance and emulsifier concentration on the distributions of gallic acid, propyl gallate, and $\alpha$-tocopherol in corn oil emulsions. Journal of Colloid and Interface Science, 389(1), 1-9. https://doi.org/10.1016/j.jcis.2012.07.036.

Malassagne-Bulgarelli, N., \& McGrath, K. M. (2009). Dynamics of oil transfer in oil-inwater emulsions. Soft Matter, 5(23), 4804. https://doi.org/10.1039/b912742a.

McClements, D. J. (2015). Food emulsions: Principles, practices, and techniques. CRC Press.

McClements, D. J. (2018). Enhanced delivery of lipophilic bioactives using emulsions: A review of major factors affecting vitamin, nutraceutical, and lipid bioaccessibility. Food \& Function, 9(1), 22-41. https://doi.org/10.1039/C7FO01515A.

McClements, D. J., Decker, E. A., Park, Y., \& Weiss, J. (2009). Structural design principles for delivery of bioactive components in nutraceuticals and functional foods. Critical Reviews in Food Science and Nutrition, 49(6), 577-606. https://doi.org/10.1080/ 10408390902841529.

McClements, D. J., \& Rao, J. (2011). Food-grade nanoemulsions: formulation, fabrication, properties, performance, biological fate, and potential toxicity. Critical Reviews in Food Science and Nutrition, 51(4), 285-330. https://doi.org/10.1080/10408398. 2011.559558.

Medina, I., Lois, S., Alcántara, D., Lucas, R., \& Morales, J. C. (2009). Effect of lipophilization of hydroxytyrosol on its antioxidant activity in fish oils and fish oil-in-water emulsions. Journal of Agricultural and Food Chemistry, 57(20), 9773-9779. https:// doi.org/10.1021/jf9023867.

Meireles, M., Losada-Barreiro, S., Costa, M., Paiva-Martins, F., Bravo-Díaz, C., \& Monteiro, L. S. (2019). Control of antioxidant efficiency of chlorogenates in emulsions: Modulation of antioxidant interfacial concentrations. Journal of the Science of Food and Agriculture. https://doi.org/10.1002/jsfa.9615.

Nuchi, C. D., Hernandez, P., McClements, D. J., \& Decker, E. A. (2002). Ability of lipid hydroperoxides to partition into surfactant micelles and alter lipid oxidation rates in emulsions. Journal of Agricultural and Food Chemistry, 50(19), 5445-5449. https:// doi.org/10.1021/jf020095j.

Osborn, H. T., \& Akoh, C. C. (2004). Effect of emulsifier type, droplet size, and oil concentration on lipid oxidation in structured lipid-based oil-in-water emulsions. Food Chemistry, 84(3), 451-456. https://doi.org/10.1016/S0308-8146(03)00270-X.

Qian, C., \& McClements, D. J. (2011). Formation of nanoemulsions stabilized by model food-grade emulsifiers using high-pressure homogenization: Factors affecting particle size. Food Hydrocolloids, 25(5), 1000-1008. https://doi.org/10.1016/j.foodhyd.2010. 09.017.

Romsted, L. S. (2012). Introduction to surfactant self-assembly. Supramolecular Chemistry, 1. https://doi.org/10.1002/9780470661345.smc013.

Romsted, L. S., \& Bravo-Díaz, C. (2013). Modeling chemical reactivity in emulsions. Current Opinion in Colloid \& Interface Science, 18(1), 3-14. https://doi.org/10.1016/j. cocis. 2012.11.001.

Saupe, A., Gordon, K. C., \& Rades, T. (2006). Structural investigations on nanoemulsions, solid lipid nanoparticles and nanostructured lipid carriers by cryo-field emission scanning electron microscopy and Raman spectroscopy. International Journal of Pharmaceutics, 314(1), 56-62. https://doi.org/10.1016/j.ijpharm.2006.01.022. 
Silva, H. D., Cerqueira, M. A., \& Vicente, A. A. (2015). Influence of surfactant and processing conditions in the stability of oil-in-water nanoemulsions. Journal of Food Engineering, 167, 89-98. https://doi.org/10.1016/j.jfoodeng.2015.07.037.

Silva, R., Losada-Barreiro, S., Paiva-Martins, F., \& Bravo-Díaz, C. (2017). Partitioning and antioxidative effect of protocatechuates in soybean oil emulsions: Relevance of emulsifier concentration. European Journal of Lipid Science and Technology, 119(6), 1600274. https://doi.org/10.1002/ejlt.201600274.

Silvestre, M. P. C., Chaiyasit, W., Brannan, R. G., McClements, D. J., \& Decker, E. A. (2000). Ability of surfactant headgroup size to alter lipid and antioxidant oxidation in oil-in-water emulsions. Journal of Agricultural and Food Chemistry, 48(6), 2057-2061. https://doi.org/10.1021/jf991162l.

Sun, C., \& Gunasekaran, S. (2009). Effects of protein concentration and oil-phase volume fraction on the stability and rheology of menhaden oil-in-water emulsions stabilized by whey protein isolate with xanthan gum. Food Hydrocolloids, 23(1), 165-174. https://doi.org/10.1016/j.foodhyd.2007.12.006
Villiere, A., Viau, M., Bronnec, I., Moreau, N., \& Genot, C. (2005). Oxidative stability of bovine serum albumin- and sodium caseinate-stabilized emulsions depends on metal availability. Journal of Agricultural and Food Chemistry, 53(5), 1514-1520. https:// doi.org/10.1021/jf0486951

Wanasundara, P. K. J. P. D., \& Shahidi, F. (2005). Antioxidants: Science, technology, and applications. In F. Shahidi (Ed.). Bailey's Industrial Oil and Fat Products (pp. 431-489). New York: Wiley \& Sons.

Waraho, T., Cardenia, V., Nishino, Y., Seneviratne, K. N., Rodriguez-Estrada, M. T., McClements, D. J., \& Decker, E. A. (2012). Antioxidant effects of mono- and diacylglycerols in non-stripped and stripped soybean oil-in-water emulsions. Food Research International, 48(2), 353-358. https://doi.org/10.1016/j.foodres.2012.05. 017.

Waraho, T., McClements, D. J., \& Decker, E. A. (2011). Mechanisms of lipid oxidation in food dispersions. Trends in Food Science \& Technology, 22(1), 3-13. https://doi.org/ 10.1016/j.tifs.2010.11.003. 